\title{
ARTICLE
}

\section{A molecular catalyst for water oxidation that binds to metal oxide surfaces}

Stafford W. Sheehan ${ }^{1}$, Julianne M. Thomsen ${ }^{1}$ Ulrich Hintermair, ${ }^{1,2}$, Robert H. Crabtree ${ }^{1}$, Gary W. Brudvig ${ }^{1}$ \& Charles A. Schmuttenmaer ${ }^{1}$

Molecular catalysts are known for their high activity and tunability, but their solubility and limited stability often restrict their use in practical applications. Here we describe how a molecular iridium catalyst for water oxidation directly and robustly binds to oxide surfaces without the need for any external stimulus or additional linking groups. On conductive electrode surfaces, this heterogenized molecular catalyst oxidizes water with low overpotential, high turnover frequency and minimal degradation. Spectroscopic and electrochemical studies show that it does not decompose into iridium oxide, thus preserving its molecular identity, and that it is capable of sustaining high activity towards water oxidation with stability comparable to state-of-the-art bulk metal oxide catalysts.

\footnotetext{
${ }^{1}$ Department of Chemistry, Yale University, 225 Prospect Street, PO Box 208107, New Haven, Connecticut 06520-8107, USA. ${ }^{2}$ Centre for Sustainable Chemical Technologies, University of Bath, Claverton Down BA2 7AY, UK. Correspondence and requests for materials should be addressed to S.W.S. (email: stafford.sheehan@yale.edu) or to U.H. (email: u.hintermair@bath.ac.uk) or to G.W.B. (email: gary.brudvig@yale.edu).
} 
E conomic and environmental concerns raised by the extensive use of fossil fuels have made alternative energy sources more attractive ${ }^{1}$. Renewable sources are particularly promising owing to their environmental sustainability and potential for widespread availability. However, there are a number of problems that need to be addressed before renewable energy can be used on a global scale ${ }^{2}$. Chief among these is the lack of reliable methods of concentrating and storing energy on a large scale, since numerous renewable energy sources, including solar and wind, are intermittent and diffuse. Towards this end, the generation of renewable fuels, in which electricity generated by renewable means is stored in the chemical bonds of a suitable fuel, has become a critically important area of research ${ }^{3}$. In one scheme for the formation of renewable fuels that mimics photosynthesis in plants, protons and electrons are extracted from water by electrochemical oxidation to be used for fuel formation, liberating $\mathrm{O}_{2}$ as a byproduct ${ }^{4}$. The efficient generation of such renewable fuels, therefore, necessitates the development of efficient, fast and stable water-oxidation catalysts (WOCs).

Of the extensive library of available WOCs, molecular species show promise because of their high activity and tunability, as well as their ability to be integrated into sophisticated molecular assemblies $^{5-12}$. Their major drawback is their limited stability, with the best homogeneous systems providing turnover numbers in the thousands ${ }^{13-15}$ to tens of thousands ${ }^{16}$. This problem is particularly pronounced for electrode-driven WOCs, which often decompose to less active materials under moderate applied potentials ${ }^{17,18}$. Building on the success of different heterogenization strategies for homogeneous catalysts in organometallic, inorganic and surface chemistry ${ }^{19-27}$, immobilization of molecular WOCs on electrode surfaces has been sought to overcome this ${ }^{28-30}$. However, in the case of an electrode-driven WOC, the ligand anchoring the catalyst to the electrode surface must display a high degree of oxidative stability, which is not always the case ${ }^{31}$. Methods that alter the electrode surface, including deposition of coating layers of $\mathrm{TiO}_{2}$ after catalyst adhesion have been shown to assist in solving this issue ${ }^{32}$. However, to date, no system has fully succeeded in combining the high efficiency and tunability of a molecular catalyst that contains a single, well-defined catalytically active site (single-site) ${ }^{33}$ with the durability and stability of a bulk material in a heterogeneous electrocatalyst for water oxidation.

In a recent report ${ }^{34}$, we identified highly active homogeneous WOCs that are formed by the oxidative removal of $\mathrm{Cp}^{*}$ (pentamethylcyclopentadienyl, $\mathrm{C}_{5} \mathrm{Me}_{5}^{-}$), an organic placeholder ligand, from well-studied $C \mathrm{p}^{*} \mathrm{Ir}$ based precursors ${ }^{35,36}$. The compounds that form from these precatalysts all possess a single bidentate chelate ligand ${ }^{37}$ per iridium that is oxidatively stable and prevents the formation of $\mathrm{IrO}_{x}$-based films ${ }^{38}$ or nanoparticles $^{39}$ under oxidative conditions. In contrast, $\mathrm{Cp}^{\star} \mathrm{Ir}$ precursors lacking a stable bidentate ligand anodically deposit amorphous $\mathrm{IrO}_{x}$ on electrodes to give a heterogeneous WOC referred to as 'blue layer' $(\mathrm{BL})^{38}$. This $\mathrm{BL}$, hydrated $\mathrm{IrO}_{x}$ nanoclusters ${ }^{40}$, and the homogeneous WOCs formed by the oxidative activation of our organometallic iridium precursors 41,42 all display a characteristic deep blue colour in their oxidized form, owing to an absorption feature near $600 \mathrm{~nm}$. This has caused confusion about the identity of the active species in these systems ${ }^{34,39}$; thus, in the following we refer only to the activated form of the molecular iridium catalyst as the hom-WOC.

In this study, we report the heterogenization of the hom-WOC to form a surface-bound, ligand-modified iridium electrocatalyst for water oxidation in acidic solutions. On self-adhering to the surface of a metal oxide at room temperature, a molecular monolayer of the catalyst is formed, which possesses higher activity than the bulk material analogue, $\mathrm{IrO}_{x}$. We show that this heterogenized molecular catalyst remains bound to the surface after extended use, eliminating the need for any linking moieties, while retaining its molecular identity and ligand-based tunability.

\section{Results}

Catalyst preparation and heterogenization. In the pursuit of combining the high efficiency of the molecular hom-WOC with the stability of bulk metal oxides, we found that when an oxide material is immersed in an aqueous solution of the hom-WOC, the material rapidly and irreversibly chemisorbs some of the bluecoloured complex from the solution (we refer to this supported heterogeneous complex as the het-WOC). The dinuclear structures shown in Fig. 1 are based on the characterization data reported in this paper and on the prior work on the homogeneous analogue (hom-WOC) ${ }^{34,41}$. These structures are consistent with the available data, but are not intended to be definitive.

In contrast, the hom-WOC does not bind to noble metals that do not form a native oxide layer such as $\mathrm{Au}$ or $\mathrm{Pt}^{41}$. To further probe the electrochemical and spectroscopic properties of the hetWOC, we moved to high surface area transparent conductive electrodes consisting of mesoporous films of tin-doped indium oxide nanoparticles (nanoITO) ${ }^{43}$ on fluorine-doped tin oxide (FTO)-coated glass slides. A solution of the hom-WOC bearing a 2-(2'pyridyl)-2-propanolate (pyalc) bidentate ligand, previously characterized as $\left[\operatorname{Ir}(\text { pyalc })\left(\mathrm{H}_{2} \mathrm{O}\right)_{2}(\mu-\mathrm{O})\right]_{2}^{2+}$, was prepared from $\left[\mathrm{Cp}^{\star} \operatorname{Ir}(\right.$ pyalc $\left.) \mathrm{OH}\right]$ and $\mathrm{NaIO}_{4}$ using established methods ${ }^{34}$. On immersion, catalyst binding to the nanoITO surface is rapid, selflimiting and does not require any external driving force such as photons or an applied potential (Fig. 2a). Control experiments show that the removal of the organometallic placeholder ligand from the precursor is required for surface binding to occur in all the cases (Supplementary Figs 1-4). Formation of a molecular monolayer is complete in $2 \mathrm{~h}$ at room temperature, with negligible absorption changes being observed at later times (Fig. 2b). Even after thorough rinsing with deionized water, the catalyst is not washed off the surface. Transmission electron microscope (TEM) and scanning electron microscope (SEM) images of the electrode indicate that no nanoparticulate deposits are formed, and energydispersive X-ray spectroscopy (EDX) measurements confirm the presence of iridium on the electrode surface without any traces of iodine or sodium from the $\mathrm{NaIO}_{4}$ used to produce the hom-WOC in solution (Supplementary Figs 5-8).

The hom-WOC absorbance peak at $608 \mathrm{~nm}$ in solution blue shifts to $580 \mathrm{~nm}$ in the het-WOC formed on binding to nanoITO (Fig. 2d), both being distinct from electrodeposited $\mathrm{IrO}_{x}^{38}$. The
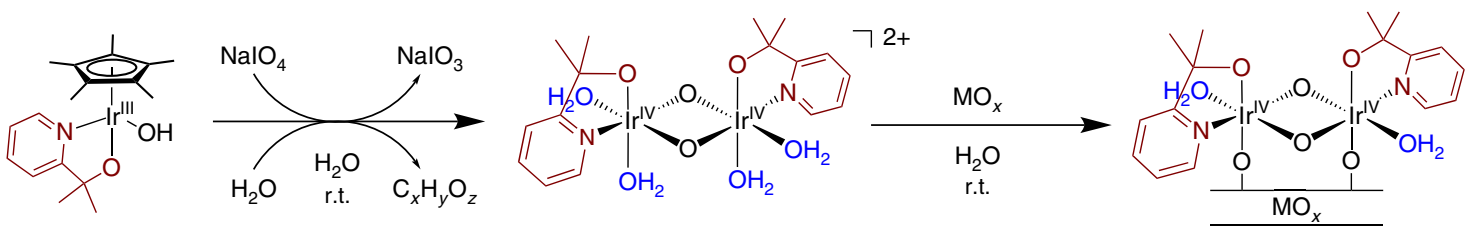

Figure 1 | Formation of the hom-WOC and proposed molecular structure for the adsorbate. Oxidation of the [Cp* $\operatorname{lr}(\mathrm{pyalc}) \mathrm{OH}]$ precursor (left) to form the $\left[\operatorname{lr}(\text { pyalc })\left(\mathrm{H}_{2} \mathrm{O}\right)_{2}(\mu-\mathrm{O})\right]_{2}^{2}+$ hom-WOC (middle) and heterogenization at room temperature (r.t.) to form the het-WOC (right) is shown. 

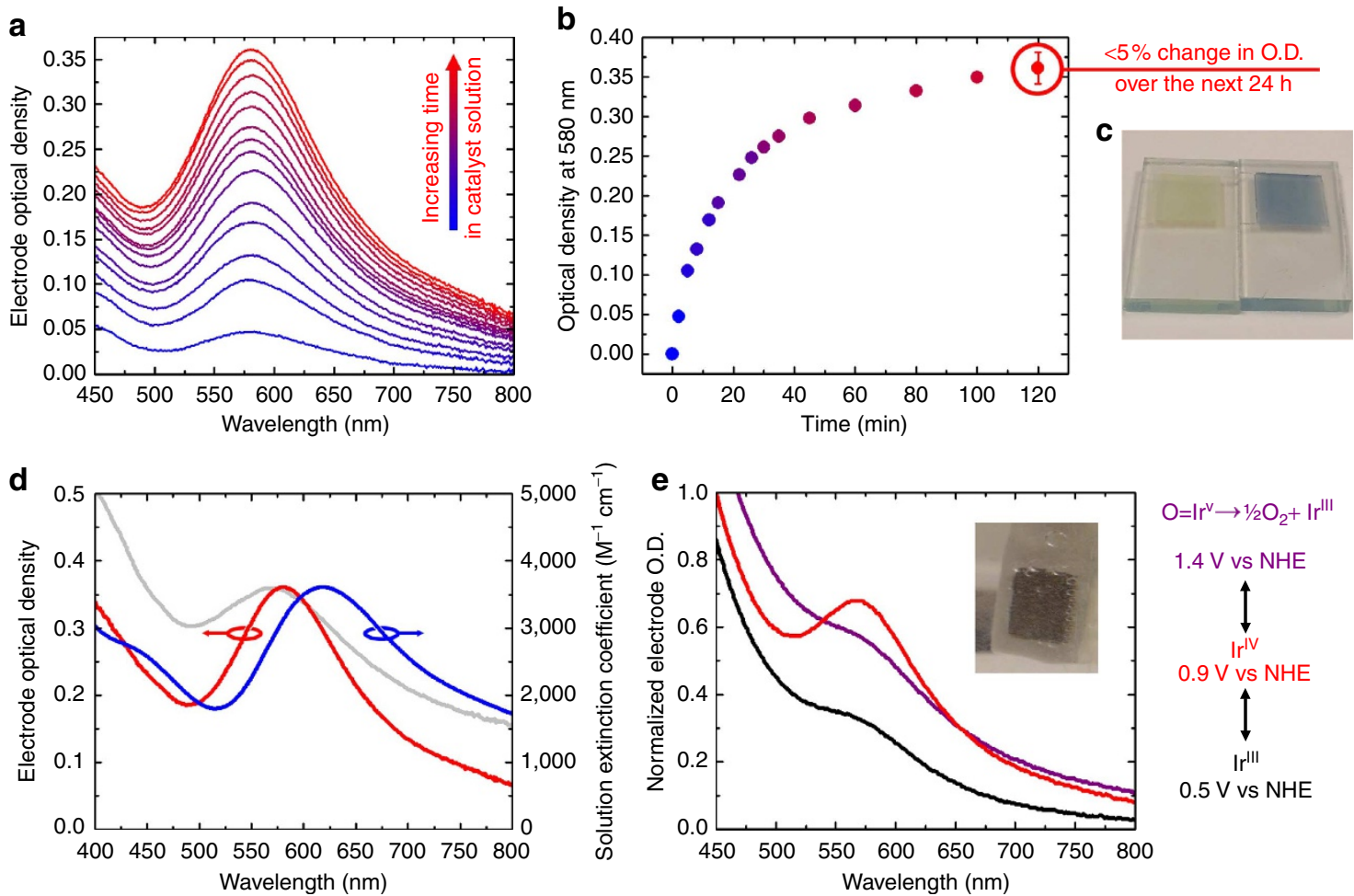

Figure 2 | Spectroscopic characterization of the het-WOC on nanolTO electrodes. (a) Optical density (O.D.) spectra of an electrode measured after increasing the amounts of time immersed in hom-WOC solution at room temperature. Between each measurement, the electrode was washed thoroughly with deionized water. (b) Increase of O.D. at $580 \mathrm{~nm}$ for the electrode as a function of immersion time, error bar shows the largest measured deviation in optical density across 3 samples after further immersion for $24 \mathrm{~h}$. (c) Photograph of an electrode before (left side of panel) and after (right side of panel) immersion in hom-WOC solution for $2 \mathrm{~h}$. (d) Comparison of the spectra of the catalyst on the surface (red) to the catalyst in solution (blue) along with $\mathrm{rr}_{x}$ electrodeposited on nano/TO (grey). (e) Spectroelectrochemical response of the electrode showing reversible transitions between Ir ${ }^{\mathrm{III}}$ (black) and Ir ${ }^{\mathrm{IV}}$ (red) oxidation states, as well as under turnover conditions (purple). The absorption feature at $580 \mathrm{~nm}$ is characteristic of $\mathrm{Ir}^{\mathrm{IV}}$, and the highly simplified legend on the right shows the potentials applied to reach these oxidation states. Inset is a photograph of the electrode under turnover conditions (1.4 $\mathrm{V}$ versus $\mathrm{NHE}$ $\mathrm{pH}$ 2.6) corresponding to the spectrum in purple.

shift that occurs during catalyst heterogenization is similar to that previously observed for the reversible deprotonation of bound water ligands ${ }^{34}$. Along with the fact that the catalyst remains bound to the surface after repeated washing, this suggests chemical binding rather than mere physisorption. Both the $\mathrm{pH}$ and the concentration of iodate in the hom-WOC solution have pronounced effects on the rate of binding (Supplementary Fig. 9). As the $\mathrm{pH}$ of the solution is decreased, the rate of catalyst binding increases. Similarly, when the $\mathrm{pH}$ of the solution is increased, the rate of catalyst binding decreases, demonstrating that the catalyst binds faster when retaining aqua ligands rather than more strongly coordinating hydroxo ligands, consistent with a water displacement mechanism. These sites are also rapidly exchanged with the anion present in solution, iodate, which can also act as a ligand ${ }^{44}$. Consistently, we found that an increase in iodate concentration inhibits surface binding; however, a low concentration of iodate is required for heterogenization. When $\mathrm{Cp}^{*} \mathrm{Ir}$ precatalyst activation to form the hom-WOC is performed electrochemically ${ }^{41}$, surface binding does not occur unless iodate is present in the solution. Electron paramagnetic resonance spectroscopy of the het-WOC showed no signals that would be expected for monomeric Ir $^{\mathrm{IV}}$ species on the surface ${ }^{45}$, while X-ray photoelectron spectroscopy (XPS) proves that iridium is indeed present in the $\operatorname{Ir}^{\mathrm{IV}}$ state (Supplementary Fig. 10) ${ }^{46-48}$. This is also the case for the hom-WOC in solution, indicating that the catalyst is still in dimer form when bound to the surface.

The absorbance peak at $580 \mathrm{~nm}$ is still evident after the electrode is immersed in aqueous solution in a spectroelectrochemical cell (Fig. 2e, Supplementary Figs 11-13). In this experiment, the catalyst-coated nanoITO working electrode forms a circuit with an $\mathrm{Ag} / \mathrm{AgCl}$ reference electrode and a platinum counter electrode. We then vary the potential applied to the working electrode to induce reversible changes in the oxidation state of iridium in the het-WOC while collecting ultraviolet-visible spectra. Importantly, the catalyst remains bound to the electrode not only in its native $\mathrm{Ir}^{\mathrm{IV}}$ state, but also in its reduced oxidation state, presumably IrII, as well as the catalytically active state, presumably $\mathrm{Ir}^{\mathrm{V}}$, from which oxygen evolution is observed 49,50 .

The stability and versatility of the het-WOC is shown by its irreversible adhesion when exposed both to acidic and basic aqueous solutions ( $\mathrm{pH}$ values ranging from 1-12) and to numerous organic solvents, including $\mathrm{CH}_{2} \mathrm{Cl}_{2}$ and $\mathrm{MeCN}$. Only repeated washing under highly alkaline conditions $(\mathrm{pH}>13)$ was found to remove the het-WOC from nanoITO, resulting in a clean electrode surface. The catalyst also adheres rapidly to different nanostructured metal oxides that are commonly used as photoanodes for light-driven water oxidation, including $\mathrm{TiO}_{2}$ and $\mathrm{WO}_{3}$ (Supplementary Fig. 14) $)^{51,52}$.

Water-oxidation performance and stability. The het-WOC maintains its activity for oxygen evolution with chemical oxidants (Fig. 3a, Supplementary Fig. 15) compared with that previously observed $^{34}$ for the hom-WOC in solution. Most importantly, however, the het-WOC shows exceptional activity when driven electrochemically. Cyclic voltammograms (CVs) in an oxygensaturated solution of $0.1 \mathrm{M} \mathrm{KNO}_{3}$ in water at $\mathrm{pH} 2.6$ (Fig. 3b, 

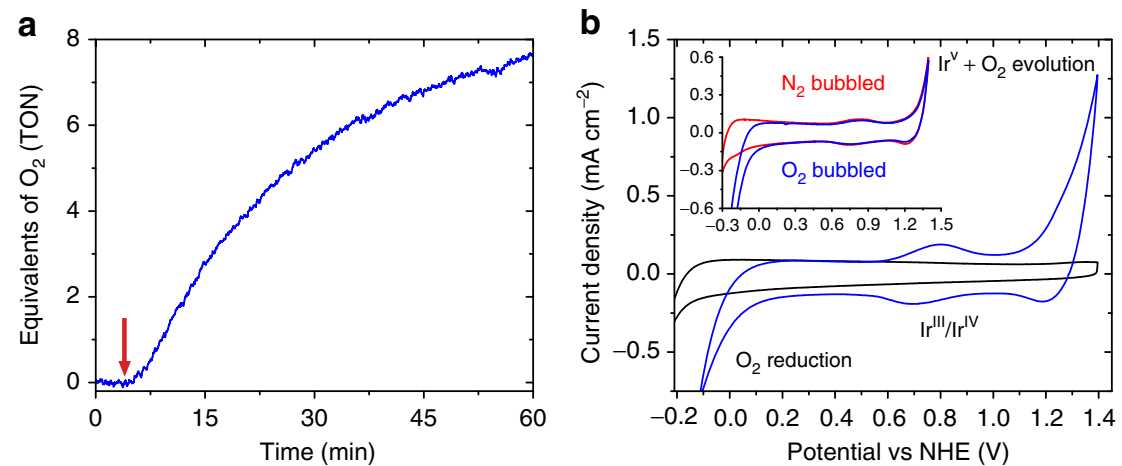

Figure 3 | Water oxidation using the het-WOC. (a) Water oxidation using $\mathrm{NalO}_{4}$ as a sacrificial oxidant with the catalyst bound to nanolTO; the red arrow corresponds to injection of $\mathrm{NalO}_{4}$ solution, initiating catalysis as quantified by the turnover number (TON) of $\mathrm{O}_{2}$ per iridium atom on the mesoporous surface. (b) CVs of a catalyst-loaded nanolTO electrode (blue) compared with a similar nanolTO electrode without catalyst (black) in an oxygen-saturated solution of $0.1 \mathrm{M} \mathrm{KNO}_{3}$ in water at $\mathrm{pH} 2.6$, taken with a $10 \mathrm{mVs}^{-1}$ scan rate. Inset shows the effect of saturating $\mathrm{O}_{2}$ or $\mathrm{N}_{2}$ gas in solution when using thinner electrodes, highlighting the catalytic wave for oxygen reduction at $\mathrm{O} V$ versus NHE.

Supplementary Fig. 16) show reversible $\mathrm{Ir}^{\mathrm{III}} / \mathrm{Ir}^{\mathrm{IV}}$ charging features with $\mathrm{E}_{1 / 2}=0.75 \mathrm{~V}$ versus the normal hydrogen electrode (NHE), as well as reversible water oxidation/oxygen reduction similar to traditional iridium oxides, but lacking a redox feature that has been assigned to the oxidation of $\mathrm{Ir}^{\mathrm{IV}}$ to $\mathrm{Ir}^{\mathrm{V}}$ (ref. 53). The onset of the water-oxidation catalytic wave occurs at a distinctively lower potential than the $\mathrm{Ir}^{\mathrm{IV}} / \mathrm{Ir}^{\mathrm{V}}$ redox couple in $\mathrm{IrO}_{x}$ samples prepared by different means ${ }^{54}$ and, thereby, obscures the $\mathrm{Ir}^{\mathrm{IV}} / \mathrm{Ir}^{\mathrm{V}}$ charging feature. Drawing a parallel to previously suggested mechanisms for Ir-catalysed water oxidation $^{36,49,50}$, we postulate that this is a direct result of this catalyst's highly active $\operatorname{Ir}^{\mathrm{V}}$ state, which along with the high electroactivity of the molecular iridium compound on the surface, allows for water oxidation at low overpotentials. Specifically, integration of the $\mathrm{Ir}^{\mathrm{III}} / \mathrm{Ir}^{\mathrm{IV}}$ wave and comparison with the total iridium loading derived from ultraviolet-visible measurements demonstrates that $>90 \%$ of iridium on the electrode is electroactive, as is expected for a molecular monolayer ${ }^{55}$.

Previously, we reported that the organometallic precursor complexes used to form the hom-WOCs by oxidative activation do not show any activity for electrode-driven water oxidation ${ }^{41}$. We also find that they do not self-adhere to oxide surfaces. Comparing both the activated forms, the kinetics of water oxidation appear to be different between the hom-WOC and the het-WOC: the H/D kinetic isotope effect (KIE) of 2.01 for the hom-WOC differs significantly from the KIE of 1.0 found for the het-WOC, when run at potentials below the appearance of masstransport related effects at the electrode surface (Supplementary Fig. 17). This suggests that different rate-limiting steps are applied to each. The KIE of unity for the het-WOC may indicate that the rate-determining step is electron transfer from the Ir centres in the catalyst to the metal oxide scaffold, rather than any step involving water. KIEs that are close to 1 for similar reasons have been seen for iridium oxide colloids and related materials ${ }^{36,56}$.

In comparison with bulk $\mathrm{IrO}_{x}$ species, one advantage of using single-site surface-bound molecular catalysts for water oxidation is accurate control of electrode overpotential by tuning the scaffold surface area, thereby changing catalyst loading. By increasing the nanoporous film's thickness, the overpotential of the electrode at specific current densities can be decreased (Fig. 4). For example, typical 3- $\mu$ m-thick nanoITO films require an overpotential of $275 \mathrm{mV}$ to attain a catalytic current of $0.5 \mathrm{~mA} \mathrm{~cm}^{-2}$, whereas $18-\mu \mathrm{m}$-thick films require $<160 \mathrm{mV}$. Although limited tunability of the number of active sites in bulk or nanostructured WOCs prevents direct comparison, we are not aware of any lower overpotential values reported in the literature

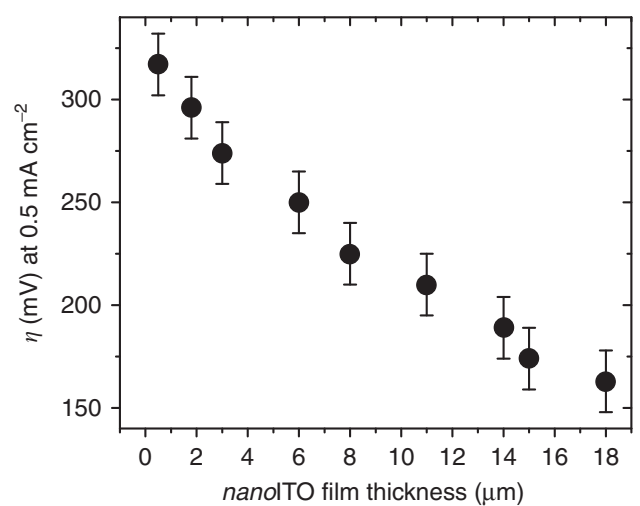

Figure 4 | Electrode overpotential as a function of nanoporous film thickness. The film thickness is directly proportional to catalyst loading. As we increase the catalyst loading on the electrode, the overpotential required to reach $0.5 \mathrm{~mA} \mathrm{~cm}^{-2}$ decreases. Error bars reported represent the largest s.d. (sample size of five electrodes) across the nine film thicknesses measured. Data were gathered using 5-min dwell times to allow the electrodes to adequately stabilize.

for this current density. Standardized benchmarking experiments comparing the het-WOC to $\mathrm{IrO}_{x}$ show that the het-WOC possesses a lower overpotential for water oxidation in all cases (Supplementary Fig. 18) ${ }^{57}$. However, any comparison between single-site molecular species and bulk heterogeneous catalysts is complicated because of the difficulty of determining the turnover rates per metal atom in bulk materials needed to accurately gauge the relative activity on a fair basis.

To further investigate the het-WOC mechanistically, Tafel plots of catalytic currents were made over a range of $\mathrm{pH}$ and buffer conditions (Fig. 5a) and with electrodes of varying thickness of the porous nanoITO film to increase catalyst loading by increasing the electrode surface area (Fig. 5b). Limitations on proton diffusion through the nanoporous films on electrodes ${ }^{58,59}$ cause a decrease in measured activity due to the low buffering capacity of $\mathrm{KNO}_{3}$ at $\mathrm{pH}$ 7. As thicker nanoITO films are used, the $\mathrm{pH}$ gradient formed through the film decreases electrode performance over time regardless of the catalyst's stability, because the locally generated highly acidic conditions etch the ITO support ${ }^{60}$. The use of a buffer may inhibit this effect, and in the presence of phosphate the het-WOC behaves in a manner identical to $\mathrm{IrO}_{x}$ materials ${ }^{61}$. Along with similarities in their $\mathrm{pH}$ dependence (Supplementary Fig. 19), CVs and spectroelectrochemical measurements, these results support the 

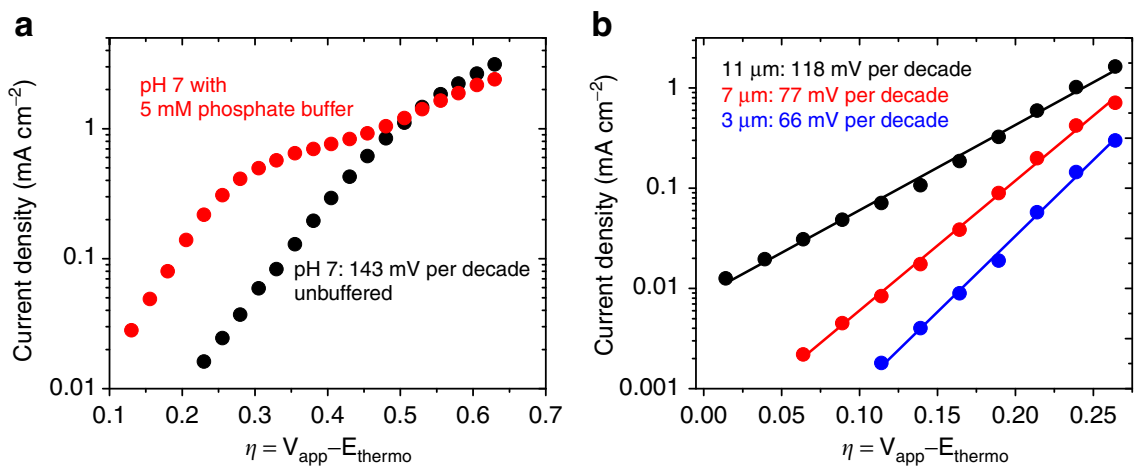

Figure 5 | Tafel plots for the het-WOC on nanorTO. (a) Tafel plots at $\mathrm{pH} 7$ showing the effect of adding a phosphate buffer to the $0.1 \mathrm{M} \mathrm{KNO}_{3}$ solution. (b) Tafel plots at $\mathrm{pH} 2.6$ without any added buffer. A decrease in Tafel slope $(\Delta \eta / \Delta \log (i))$ with decreasing film thickness corresponds to a decrease in diffusion-related $\mathrm{pH}$ effects.
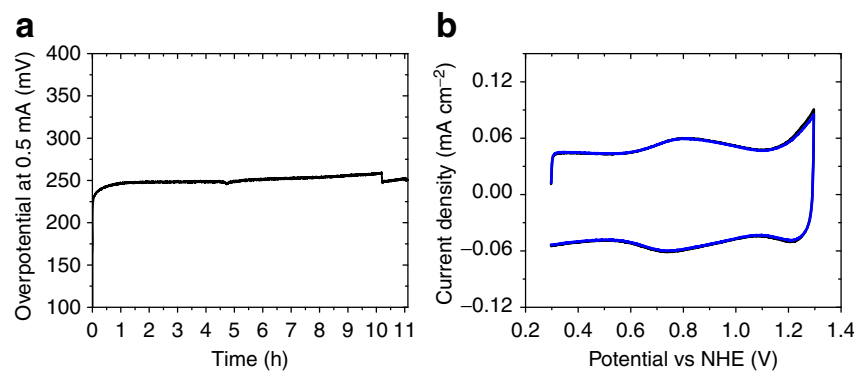

Figure 6 | Stability for electrochemical water oxidation. (a)

Chronopotentiometry showing stability of the het-WOC during sustained water oxidation for over $11 \mathrm{~h}(\mathrm{pH} 2.6)$. Small increases and decreases correspond to oxygen bubble build-up and release on the surface, which was minimized by rapidly stirring the solution. (b) $\mathrm{CVs}\left(\mathrm{pH} 2.6,10 \mathrm{mVs}^{-1}\right.$ scan rate) after $1 \mathrm{~h}$ of electrolysis (black) and after $>12 \mathrm{~h}$ of electrolysis (blue) using electrodes thin enough to mitigate local pH effects, showing full preservation of the electrode characteristics after sustained use.

hypothesis that the active catalytic sites in iridium oxides such as $\mathrm{BL}$ are mechanistically similar to those in the het-WOC. At a pH of 2.6, where $\mathrm{pH}$ is less sensitive to proton production from water oxidation, Tafel slopes increase as nanoITO film thickness is increased since the protons generated from water oxidation must diffuse through a thicker film. Remarkably, however, as seen in Fig. 5b, 11- $\mu \mathrm{m}$-thick samples have high enough catalyst loading to allow sustained current densities with an onset of linearity in the Tafel plot beginning at overpotentials as low as $14 \mathrm{mV}$ (where the current density is $11 \mu \mathrm{Acm}^{-2}$ ). This is consistent with the onset of water oxidation $\left(E_{\text {cat }}\right)$ for this catalyst being at nearly the thermodynamic potential, though the current is below the threshold of $0.5 \mathrm{~mA} \mathrm{~cm}^{-2}$ required for practical use.

The het-WOC also shows excellent stability, and is capable of sustaining water oxidation for many hours at a $250 \mathrm{mV}$ overpotential without degradation (Fig. 6a, Supplementary Figs 20-22), reaching turnover numbers in excess of $10^{6} \mathrm{O}_{2}$ evolved per iridium atom over multiple trials, as calculated by measuring the current passed through the electrode assuming a four-electron oxidative process. CVs of the electrode during and after these stability tests confirm that there is minimal loss in catalyst on the electrode surface (Fig. 6b). Moving to higher applied potentials $(+520 \mathrm{mV}$ relative to thermodynamic) we measure a turnover frequency of $7.9 \mathrm{~s}^{-1} \mathrm{O}_{2}$ molecules evolved per electroactive iridium atom, which is one of the highest values reported to date. In addition, a $99 \%$ Faradaic yield is measured for $\mathrm{O}_{2}$ evolution over $2 \mathrm{~h}$ using a phase fluorometric oxygen sensor (Supplementary Figs 23 and 24). To compare this with benchmark iridium oxide nanomaterials reported in the literature, films of $2 \mathrm{~nm} \mathrm{IrO}_{x}$ clusters with comparable electroactivity require an overpotential of $680 \mathrm{mV}$ to achieve a turnover frequency of $6.0 \mathrm{~s}^{-1}$, while larger $60-100 \mathrm{~nm} \mathrm{IrO}_{2}$ nanoparticles having $16 \%$ electroactivity require $580 \mathrm{mV}$ to achieve a rate of $6.6 \mathrm{~s}^{-1} \mathrm{O}_{2}$ molecules evolved per electroactive iridium atom ${ }^{53,62}$. The observed high performance and atomic efficiency further distinguishes this molecular iridium WOC from traditional bulk iridium oxides.

To further probe the molecular nature of the het-WOC, we compare it with iridium oxide-based materials formed by heating an as-prepared electrode to 500 or $700^{\circ} \mathrm{C}$ for $1 \mathrm{~h}$ (Fig. 7a). Scanning TEM analysis coupled with EDX mapping (STEMEDX) displays the nanoscale coverage of iridium on ITO nanoparticles. As deposited, there is a highly conformal coating of iridium on each particle, consistent with a surface-bound molecular monolayer (Fig. 7b, Supplementary Fig. 25). The corresponding $\mathrm{CV}$ has a catalytic wave for water oxidation beginning at $1.1 \mathrm{~V}$ versus $\mathrm{NHE}$ at $\mathrm{pH} 2.6$ (1.25 V versus RHE). Heating an electrode covered with the molecular catalyst at $500^{\circ} \mathrm{C}$ in air burns off the pyalc ligand without affecting conformal coating of iridium oxide around each ITO nanoparticle (Fig. 7c, Supplementary Figs 26 and 27). This causes an anodic shift in the catalytic wave for water oxidation to $1.3 \mathrm{~V}$ versus NHE, revealing a feature at $1.1 \mathrm{~V}$ versus NHE typically assigned to the $\mathrm{Ir}^{\mathrm{IV}} / \mathrm{Ir}^{\mathrm{V}}$ redox couple 33,54 ; in the unheated sample, the catalytic wave for water oxidation obscures this feature. Heating an electrode coated with the molecular catalyst to $700{ }^{\circ} \mathrm{C}$ in air results in the formation of crystalline rutile $\mathrm{IrO}_{2}$ clusters with $\sim 20 \mathrm{~nm}$ diameter (Fig. 7d, Supplementary Fig. 28). In accordance with literature precedent ${ }^{63}$, these show even lower activity for water oxidation, in part because most of the iridium is no longer in contact with water, reducing the number of active surface sites.

Molecular nature of the het-WOC. Aside from the monolayer distribution of active iridium, the oxidatively resistant bidentate pyalc ligand bound to iridium in the het-WOC represents another striking difference between single-site molecular catalysts such as this and traditional iridium oxide materials. Therefore, it is important to show that the ligand remains after extended periods of electrolysis, demonstrating that the het-WOC is stable. XPS, thus, serves to confirm the molecular structure and stability of the catalyst on the electrode, showing that the pyalc ligand is still present after $\sim 16 \mathrm{~h}$ of electrolysis corresponding to $>100,000$ turnovers of $\mathrm{O}_{2}$ per iridium atom (Fig. 8, Supplementary Fig. 29, Supplementary Table 1). From these data, we can also conclude that the resting state for the catalyst on the 
a

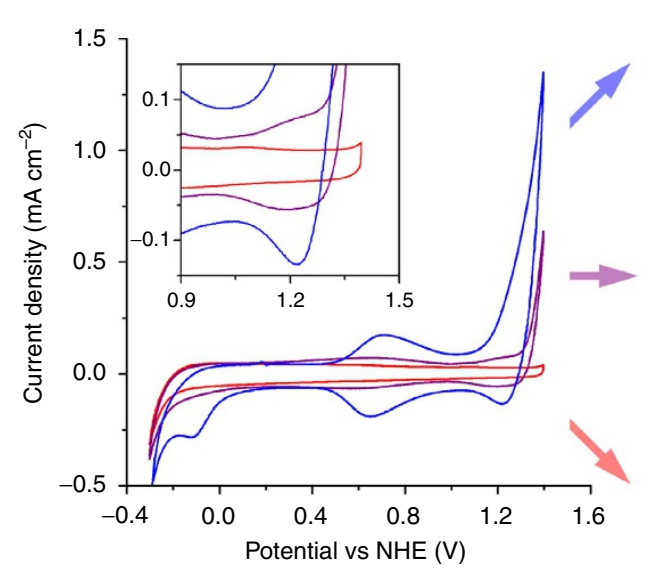

b

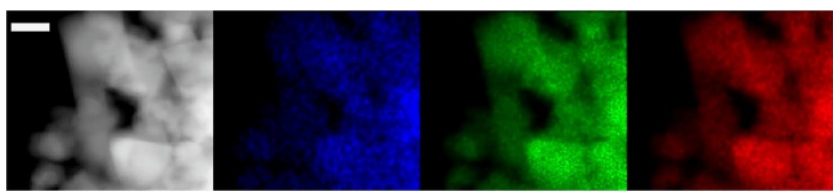

C
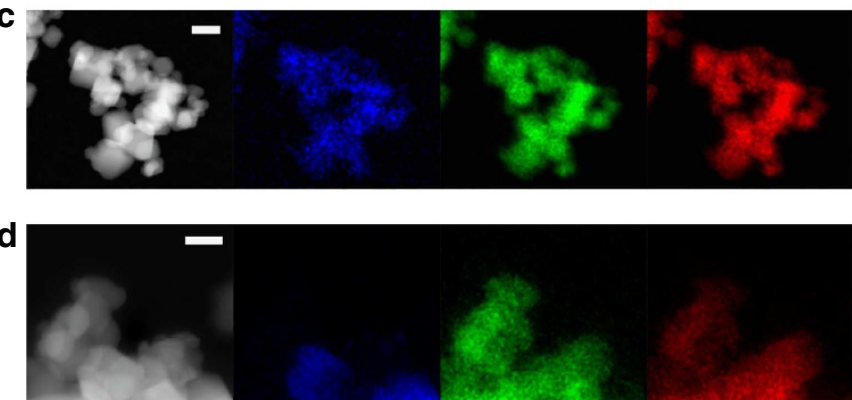

Figure 7 | Comparison of iridium oxide catalysts on nanolTo films. (a) $\mathrm{CVs}$ taken with a $10 \mathrm{mVs}^{-1} \mathrm{scan}$ rate at pH 2.6 of a catalyst-coated nanolTO electrode as-prepared (blue), heated to $500{ }^{\circ} \mathrm{C}$ (purple) and heated to $700^{\circ} \mathrm{C}$ (red). Inset is the $0.5-0.9 \mathrm{~V}$ versus NHE region expanded to more easily compare the features in the purple and red traces. (b) STEM-EDX maps of electrode material as-prepared: high-angle annular dark field image (grey), iridium detected shown in blue, indium in green and tin in red. (c) STEM-EDX map after heating to $500^{\circ} \mathrm{C}$ showing iridium remains coated around the ITO nanoparticle scaffold after the pyalc ligand is burned off (X-ray photoelectron spectra shown in Supplementary Fig. 27). (d) Corresponding maps after heating to $700^{\circ} \mathrm{C}$, the iridium is now localized in specific regions indicating nanoparticle formation (scale bars, $20 \mathrm{~nm}$ ).

a
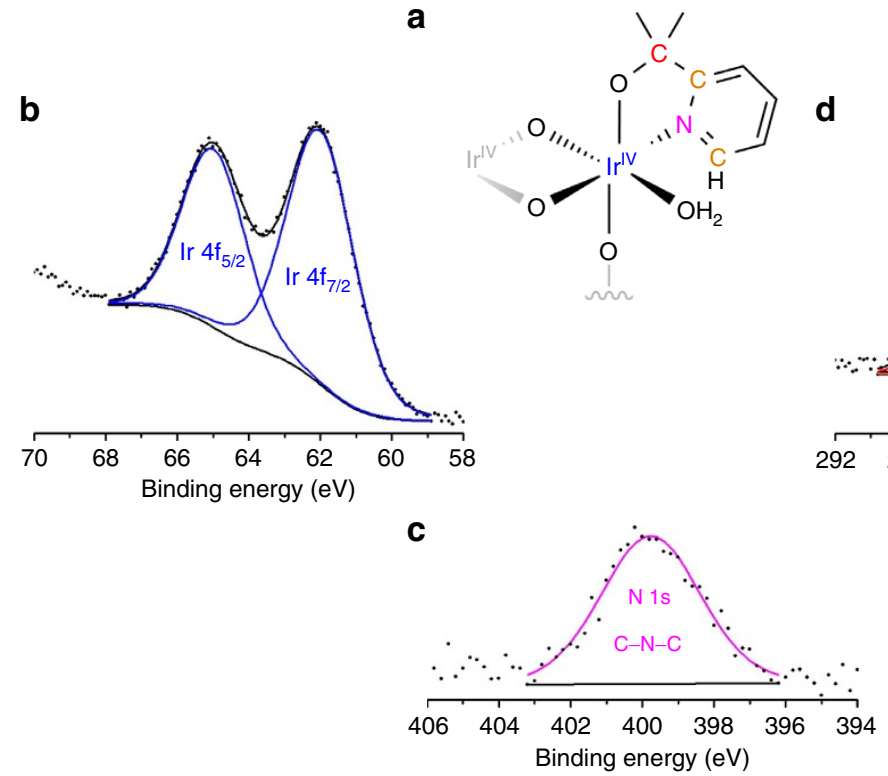

d

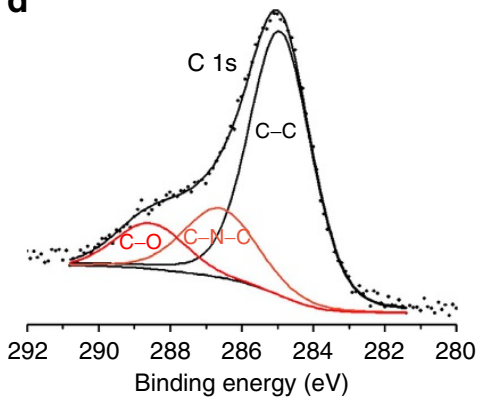

Figure 8 | X-ray photoelectron spectra taken after $\mathbf{1 6} \mathbf{h}$ of water-oxidation catalysis. Representative colour-coded schematic (a) showing elements present. Signals corresponding to the iridium (b), nitrogen (c) and carbon (d) show that both the metal and ligand are still intact after prolonged electrolysis. No changes are observed on the same film before and after electrolysis.

electrode surface involves $\operatorname{Ir}^{\mathrm{IV}}$ with a 1:1 ratio of pyalc to $\mathrm{Ir}$ as found previously for the hom-WOC in solution ${ }^{34}$.

Molecular catalysts are tunable by the variation of their ligands, and the het-WOC behaves in the same way. By changing the bidentate chelating ligand in the hom-WOC before deposition on the electrode surface, the properties of the het-WOC-functionalized electrode can be drastically changed. If, for example, $\left[\mathrm{Cp}^{\star} \operatorname{Ir}(\mathrm{bpy}) \mathrm{OH}\right] \mathrm{BF}_{4}$ bearing the 2,2'-bipyridine (bpy) ligand instead of pyalc is used as a precatalyst to form the active catalyst in solution ${ }^{34}$, a semitransparent yellow iridium compound (Supplementary Fig. 30) is deposited, having electrochemical properties that are very distinct from those of the pyalc-derived het-WOC (Supplementary Fig. 31), with much lower oxygenevolution activity being observed (Supplementary Fig. 32). We find similar differences in activity in both electrochemically and chemically driven oxygen evolution for these two hom-WOCs in solution $^{41}$. This shows that our heterogenization strategy preserves the ligand-based tunability of the hom-WOC.

\section{Discussion}

These results provide a framework for assembling surface-bound molecular catalysts with a variety of direct-binding schemes ${ }^{64,65}$. The mild conditions of deposition are particularly promising with regards to nanostructured electrode materials that are difficult to functionalize by other means ${ }^{66}$. The higher activity and stability of the described materials over previously reported heterogenized molecular WOCs ${ }^{67,68}$ shows that direct surface binding is a valid approach to attaching WOCs to electrodes. The present system also outperforms heterogeneous $\mathrm{IrO}_{x}$ materials such as $\mathrm{BL}$ as a 
WOC, although they do share similar characteristics, and our further studies will determine how this molecular species mechanistically relates to the active sites in bulk iridium oxides $^{38,69}$. The het-WOC requires minimal iridium to efficiently oxidize water relative to $\operatorname{IrO}_{x}$ catalysts, and although water-oxidation catalysis on a global scale as required for solar fuel production will likely require the use of catalysts made from more abundant elements, it is still advantageous to develop WOCs based on rare but highly active metals ${ }^{70}$. Nevertheless, this demonstration of a robust and highly efficient iridium-based molecular heterogeneous catalyst provides a new architecture for molecular heterogeneous catalysts and opens up this field to develop WOCs made from abundant materials using similar design principles.

\section{Methods}

General procedures. All the chemicals were purchased from major suppliers and used as received. Synthesis of the precatalyst [ $\left(\eta^{5}\right.$-pentamethylcyclopentadieny1) $\mathrm{Ir}^{\mathrm{III}}(2-(2$ 'pyridyl)-2-propanolate- $\mathrm{\kappa O}, \kappa \mathrm{N}) \mathrm{OH}$ ] was performed using a published procedure $^{39}$ and its activated form, the proposed $\left[\operatorname{Ir}(\text { pyalc })\left(\mathrm{H}_{2} \mathrm{O}\right)_{2}(\mu-\mathrm{O})\right]_{2}^{2+}$ compound, was synthesized by the oxidation of the precatalyst with 100 equivalents of $\mathrm{NaIO}_{4}$ (Acros Organics, 99\%) following previously published methods ${ }^{34}$.

Electrode preparation. NanoITO electrodes were prepared by spin coating (Headway PWM32 Spin Coater, Headway Research Inc.) a solution of ITO nanoparticles (Sigma-Aldrich, $<50 \mathrm{~nm}$ particle size) suspended in a $5 \mathrm{M}$ acetic acid/ethanol solution onto a $2.2 \mathrm{~mm}$-thick FTO-coated glass slide (FTO, TEC 7, Hartford Glass Co. Inc.), followed by heating at $500{ }^{\circ} \mathrm{C}$ in air for $1 \mathrm{~h}$, cooling to room temperature, then heating to $300^{\circ} \mathrm{C}$ in a $3 \% \mathrm{H}_{2} / \mathrm{N}_{2}$ atmosphere for $1 \mathrm{~h}$ and cooling back to room temperature. They were immersed in a catalyst solution formed by oxidizing $10 \mathrm{mM}\left[\mathrm{Cp}^{*} \operatorname{Ir}(\right.$ pyalc) $\mathrm{OH}]$ in $30 \mathrm{ml}$ deionized water with 100 equivalents of $\mathrm{NaIO}_{4}$. The electrodes were removed after $2 \mathrm{~h}$, washed thoroughly with deionized water, and had acquired a visible blue colour. Side-by-side controls were used to measure the relative decrease in absorption of the catalyst solution, to approximately determine the amount of iridium that had adhered to the surface of the electrode. NanoITO film thicknesses were measured using a profilometer (KLA Tencor Alphastep 200). Additional preparatory procedures for electrodes made from $\mathrm{TiO}_{2}$ and $\mathrm{WO}_{3}$, as well as the procedure for determining catalyst loading are detailed in the Supplementary Methods.

Ultraviolet-visible spectroscopy. For optical studies including the data gathered in Fig. 2a,b,d, a Varian Cary 3 spectrophotometer with an integrating sphere attachment in absorption mode was used. A $6.45 \mathrm{~cm}^{2}$ geometric surface area of nanoITO on FTO-coated glass was used to completely cover the aperture of the integrating sphere. A background spectrum was taken before immersion of the substrate into the hom-WOC solution. Ultraviolet-visible spectra for $\mathrm{IrO}_{x}$ on nanoITO were taken by electrodepositing $\mathrm{IrO}_{x}$ from a solution containing $\left[\mathrm{Cp}^{*} \operatorname{Ir}\left(\mathrm{H}_{2} \mathrm{O}\right)_{3}\right] \mathrm{SO}_{4}$ using the conditions outlined in ref. 38 with 50 deposition cycles at $50 \mathrm{mV} \mathrm{s}^{-1}$ scan rate. For the data gathered in Fig. 2e, an electrochemical cell as described previously was assembled in a $1 \mathrm{~cm}^{2}$ quartz ultraviolet-visible cuvette attached to an integrating sphere in a Shimadzu UV-2600 spectrophotometer. Standard electrolyte conditions of $0.1 \mathrm{M} \mathrm{KNO}_{3}$ adjusted to $\mathrm{pH} 2.6$ were used. Catalyst-coated nanoITO electrodes that were $6.45 \mathrm{~cm}$ long and $0.7 \mathrm{~cm}$ wide were prepared by cutting an FTO-coated glass slide with a $7 \mu \mathrm{m}$ thick catalyst-coated nanoITO film to the appropriate dimensions. Working electrodes were constructed by attaching a copper wire to an exposed FTO surface on one side of the nanoITO-coated FTO slide using conductive epoxy (Fast Setting Conductive Silver Epoxy, SPI). Six hours were allotted for the conductive epoxy to cure, then non-conductive water-resistant marine epoxy (White Marine Epoxy, Loctite, $24 \mathrm{~h}$ allotted to cure) was applied on top of the conductive epoxy to prevent electrical contact between the wire leads and electrolyte, so that the only conductive component exposed was the catalyst-coated nanoITO. This was placed into the quartz cuvette along with $\mathrm{Pt}$ mesh counter and $\mathrm{Ag} / \mathrm{AgCl}$ reference (Bioanalytical Systems, Inc.) electrodes adjacent to the integrating sphere, connected to a potentiostat (WavenowXV, Pine), and chronoamperometric experiments with the potentials detailed in Fig. 2e were performed. The electrodes were given 3 min to stabilize at that potential, then a ultraviolet-visible spectrum was taken using the integrating sphere in absorption mode. A blank nanoITO-coated FTO slide without catalyst was used for a background scan.

Chemical oxidation. Oxygen was detected with a Clark electrode using a custommade zero-headspace $10 \mathrm{ml}$ glass cell, water jacketed for constant temperature. A Teflon cap through which the Clark electrode membrane contacted deionized water (adjusted to pH 2.5 using nitric acid) also held a catalyst-coated nanoITO film on FTO-coated glass sample that was submerged in the cell. Catalyst-loaded samples were prepared similarly to those used for electrochemistry, except with a $1.6 \mathrm{~cm}^{2}$ geometric surface area. The Clark electrode was allowed to stabilize while stirring the deionized water solution for $1 \mathrm{~h}$ before injection with an oxidizing solution of freshly prepared $\mathrm{NaIO}_{4}$ in deionized water. Catalyst response and $\mathrm{O}_{2}$ generation occurred immediately, without any lag phase. A typical experiment, such as is shown in Fig. $3 \mathrm{a}$, used $25 \mu \mathrm{l}$ of $0.25 \mathrm{M} \mathrm{NaIO}_{4}$ in deionized water. The oxygen content in the cell was monitored until oxygen evolution ceased, which for a loading of around $50 \mathrm{nmol}$ of iridium (corresponding to a nanoITO film $11-\mu \mathrm{m}$ thick) took $\sim 90 \mathrm{~min}$. Data were collected while stirring the solution to ensure steady state oxygen readings.

Electrochemical characterization. Electrolyte pHs were adjusted using $1 \mathrm{M}$ $\mathrm{HNO}_{3}$ or $1 \mathrm{M} \mathrm{KOH}$. Electrochemical data were taken using $0.1 \mathrm{M} \mathrm{KNO}_{3}$ in deionized water as an electrolyte, adjusted to $\mathrm{pH} 2.6$ unless stated otherwise, with an $\mathrm{Ag} / \mathrm{AgCl}$ reference electrode (Bioanalytical Systems Inc.) and Pt mesh counter electrode. Measurements were taken using a Princeton Applied Research Versastat 4-400 potentiostat in a standard three-electrode configuration. Vigorous stirring was required in unbuffered solutions during long-term experiments to prevent etching of the nanoITO electrode under acidic conditions. Long-term stability testing and oxygen detection using phase fluorometry were performed in a twochamber electrochemical cell, with working and counter electrode chambers separated by a glass frit. For these experiments, such as those shown in Fig. 6, low catalyst loading was achieved with $300-500 \mathrm{~nm}$ thickness nanoITO films on a $6.45 \mathrm{~cm}^{2}$ substrate, thereby minimizing local $\mathrm{pH}$ effects due to the low buffer capacity of $\mathrm{KNO}_{3}$ over the $\mathrm{pH}$ range studied. SEM and TEM data were taken both before and after electrolysis to determine that there were no changes to sample morphology and CVs were taken to ensure the minimal loss of electroactive catalyst over the course of an experiment. Further details on electrochemical methods and additional controls are included in the Supporting Information (Supplementary Figs 33-35).

Electron microscopy. SEM images and SEM-EDX data were taken on a Hitachi SU-70 Analytical Scanning Electron Microscope. Images of the samples were taken both before and after electrolysis. TEM images, EDX data, high-angle annular dark field images and STEM-EDX maps were taken using a FEI Tecnai Osiris TEM operating at $200 \mathrm{kV}$. Samples were prepared by scraping nanoITO off of an electrode into deionized water, then suspending them onto a silicon monoxide coated TEM grid (Ted Pella).

X-ray photoelectron spectroscopy. X-ray photoelectron spectra were collected using an $\mathrm{Al}$ anode $(h v=1486.6 \mathrm{eV})$ and a double-pass cylinder mirror analyzer (PHI 15- 255G). Geometric surface area $6.45 \mathrm{~cm}^{2}$ samples with a $\sim 400 \mathrm{~nm}$ thick nanoITO film on the surface of FTO-coated glass were used for XPS studies. All experiments used a pass energy of $35.75 \mathrm{eV}$. Spectra were calibrated to an $\mathrm{Au}$ standard, and peak fits were performed using XPSPeak (version 4.1). Additional information regarding peak fitting and experimental details for Supplementary Figures can be found in the Supplementary Methods.

\section{References}

1. Chu, S. \& Majumdar, A. Opportunities and challenges for a sustainable energy future. Nature 488, 294-303 (2012).

2. Lewis, N. S. \& Nocera, D. G. Powering the planet: chemical challenges in solar energy utilization. Proc. Natl Acad. Sci. USA 103, 15729-15735 (2006).

3. Faunce, T. et al. Artificial photosynthesis as a frontier technology for energy sustainability. Energy Environ. Sci. 6, 1074-1076 (2013).

4. Blankenship, R. E. et al. Comparing photosynthetic and photovoltaic efficiencies and recognizing the potential for improvement. Science 332, 805-809 (2011).

5. Llobet, A. Molecular Water Oxidation Catalysis: A Key Topic for New Sustainable Energy Conversion Schemes (Wiley, 2014).

6. Ruttinger, W. \& Dismukes, G. C. Synthetic water-oxidation catalysts for artificial photosynthetic water oxidation. Chem. Rev. 97, 1-24 (1997).

7. Young, K. J. et al. Light-driven water oxidation for solar fuels. Coord. Chem. Rev. 256, 2503-2520 (2012).

8. Wasylenko, D. J., Palmer, R. D. \& Berlinguette, C. P. Homogeneous water oxidation catalysts containing a single metal site. Chem. Commun. 49, 218-227 (2013).

9. Cole-Hamilton, D. J. Homogeneous catalysis-new approaches to catalyst separation, recovery, and recycling. Science 299, 1702-1706 (2003).

10. Evangelisti, F., Güttinger, R., Moré, R., Luber, S. \& Patzke, G. R. Closer to Photosystem II: $\mathrm{A} \mathrm{Co}_{4} \mathrm{O}_{4}$ Cubane Catalyst with Flexible Ligand Architecture. J. Am. Chem. Soc. 135, 18734-18737 (2013).

11. Evangelisti, F., Car, P. E., Blacque, O. \& Patzke, G. R. Photocatalytic water oxidation with cobalt-containing tungstobismutates: tuning the metal core. Catal. Sci. Technol. 3, 3117-3129 (2013).

12. Eisenberg, R. \& Gray, H. B. Preface on Making Oxygen. Inorg. Chem. 47, 1697-1699 (2008). 
13. Duan, L. L. et al. A molecular ruthenium catalyst with water-oxidation activity comparable to that of photosystem II. Nat. Chem. 4, 418-423 (2012).

14. Fillol, J. L. et al. Efficient water oxidation catalysts based on readily available iron coordination complexes. Nat. Chem. 3, 807-813 (2011).

15. Yin, Q. S. et al. A fast soluble carbon-free molecular water oxidation catalyst based on abundant metals. Science 328, 342-345 (2010).

16. Lewandowska-Andralojc, A. et al. Efficient water oxidation with organometallic iridium complexes as precatalysts. Phys. Chem. Chem. Phys. 16, 11976-11987 (2014).

17. Barnett, S. M., Goldberg, K. I. \& Mayer, J. M. A soluble copper-bipyridine water-oxidation electrocatalyst. Nat. Chem. 4, 498-502 (2012).

18. Dau, H. et al. The Mechanism of Water Oxidation: From Electrolysis via Homogeneous to Biological Catalysis. ChemCatChem 2, 724-761 (2010).

19. Wegener, S. L., Marks, T. J. \& Stair, P. C. Design strategies for the molecular level synthesis of supported catalysts. Acc. Chem. Res. 45, 206-214 (2012).

20. Muratsugu, S. \& Tada, M. Molecularly imprinted Ru complex catalysts integrated on oxide surfaces. Acc. Chem. Res. 46, 300-311 (2013).

21. Comas-Vives, A. et al. Single-site homogeneous and heterogenized gold(III) hydrogenation catalysts: mechanistic implications. J. Am. Chem. Soc. 128, 4756-4765 (2006).

22. Murray, R. W., Ewing, A. G. \& Durst, R. A. Chemically modified electrodesmolecular design for electroanalysis. Anal. Chem. 59, 379A-390A (1987).

23. Beck, J. S. et al. A new family of mesoporous molecular-sieves prepared with liquid-crystal templates. J. Am. Chem. Soc. 114, 10834-10843 (1992).

24. Muresan, N. M., Willkomm, J., Mersch, D., Vaynzof, Y. \& Reisner, E. Immobilization of a molecular cobaloxime catalyst for hydrogen evolution on a mesoporous metal oxide electrode. Angew. Chem. Int. Ed. 51, 12749-12753 (2012).

25. Soriaga, M. P. Surface Coordination Chemistry of Monometallic and Bimetallic Electrocatalysts. Chem. Rev. 90, 771-793 (1990).

26. Terry, T. J. \& Stack, T. D. P. Covalent Heterogenization of a Discrete Mn(II) Bis-Phen Complex by a Metal-Template/Metal-Exchange Method: An Epoxidation Catalyst with Enhanced Reactivity. J. Am. Chem. Soc. 130, 4945-4953 (2008)

27. Somorjai, G., Frei, H. \& Park, J. Y. Advancing the Frontiers in Nanocatalysis, Biointerfaces, and Renewable Energy Conversion by Innovations of Surface Techniques. J. Am. Chem. Soc. 131, 16589-16605 (2009).

28. Concepcion, J. J., Binstead, R. A., Alibabaei, L. \& Meyer, T. J. Application of the rotating ring-disc-electrode technique to water oxidation by surface-bound molecular catalysts. Inorg. Chem. 52, 10744-10746 (2013).

29. Zhong, D. K., Zhao, S. L., Polyansky, D. E. \& Fujita, E. Diminished photoisomerization of active ruthenium water oxidation catalyst by anchoring to metal oxide electrodes. J. Catal. 307, 140-147 (2013).

30. Klepser, B. M. \& Bartlett, B. M. Anchoring a molecular iron catalyst to solarresponsive $\mathrm{WO}_{3}$ improves the rate and selectivity of photoelectrochemical water oxidation. J. Am. Chem. Soc. 136, 1694-1697 (2014).

31. Crabtree, R. H. The stability of organometallic ligands in oxidation catalysis. J. Organomet. Chem. 751, 174-180 (2014).

32. Vannucci, A. K. et al. Crossing the divide between homogeneous and heterogeneous catalysis in water oxidation. Proc. Natl Acad. Sci. USA 110, 20918-20922 (2013).

33. Thomas, J. M., Raja, R. \& Lewis, D. W. Single-site heterogeneous catalysts Angew. Chem. Int. Ed. 44, 6456-6482 (2005).

34. Hintermair, U. et al. Precursor transformation during molecular oxidation catalysis with organometallic iridium complexes. J. Am. Chem. Soc. 135, 10837-10851 (2013)

35. Hull, J. F. et al. Highly active and robust $\left(\mathrm{Cp}^{*}\right)$ iridium complexes for catalytic water oxidation. J. Am. Chem. Soc. 131, 8730-8731 (2009).

36. Blakemore, J. D. et al. Half-sandwich iridium complexes for homogeneous water-oxidation catalysis. J. Am. Chem. Soc. 132, 16017-16029 (2010).

37. Schley, N. D. et al. Distinguishing homogeneous from heterogeneous catalysis in electrode-driven water oxidation with molecular iridium complexes. J. Am. Chem. Soc. 133, 10473-10481 (2011).

38. Blakemore, J. D. et al. Anodic deposition of a robust iridium-based wateroxidation catalyst from organometallic precursors. Chem. Sci. 2, 94-98 (2011).

39. Hintermair, U., Hashmi, S. M., Elimelech, M. \& Crabtree, R. H. Particle formation during oxidation catalysis with $\mathrm{Cp}^{*}$ iridium complexes. J. Am. Chem. Soc. 134, 9785-9795 (2012)

40. Blakemore, J. D. et al. Comparison of amorphous iridium water-oxidation electrocatalysts prepared from soluble precursors. Inorg. Chem. 51, 7749-7763 (2012).

41. Thomsen, J. M. et al. Electrochemical activation of $\mathrm{Cp}^{*}$ iridium complexes for electrode-driven water-oxidation catalysis. J. Am. Chem. Soc. 136, 13826-13834 (2014).

42. Ingram, A. J. et al. Modes of activation of organometallic iridium complexes for catalytic water and C-H oxidation. Inorg. Chem. 53, 423-433 (2014).

43. Chen, Z. F., Concepcion, J. J., Hull, J. F., Hoertz, P. G. \& Meyer, T. J. Catalytic water oxidation on derivatized nanoITO. Dalton. Trans. 39, 6950-6952 (2010).
44. Rose, D., Lever, F. M., Powell, A. R. \& Wilkinson, G. The Nature of iridium(IV) iodate. J. Chem. Soc. A 1969, 1690-1691 (1969).

45. Brewster, T. P. et al. An iridium(IV) species, $\left[\mathrm{Cp}^{\star} \operatorname{Ir}(\mathrm{NHC}) \mathrm{Cl}\right]^{+}$, related to a water-oxidation catalyst. Organometallics 30, 965-973 (2011).

46. Rubel, M. et al. Characterization of $\mathrm{IrO}_{2}-\mathrm{SnO}_{2}$ thin-layers by electron and ion spectroscopies. Vacuum 45, 423-427 (1994).

47. Wang, C., Wang, J. L. \& Lin, W. B. Elucidating molecular iridium water oxidation catalysts using metal-organic frameworks: a comprehensive structural, catalytic, spectroscopic, and kinetic study. J. Am. Chem. Soc. 134, 19895-19908 (2012).

48. Kim, Y. I. \& Hatfield, W. E. Electrical, magnetic and spectroscopic properties of tetrathiafulvalene charge-transfer compounds with iron, ruthenium, rhodium and iridium halides. Inorg. Chim. Acta 188, 15-24 (1991).

49. Minguzzi, A. et al. Observing the oxidation state turnover in heterogeneous iridium-based water oxidation catalysts. Chem. Sci. 5, 3591-3597 (2014).

50. Sanchez Casalongue, H. G. et al. In Situ Observation of Surface Species on Iridium Oxide Nanoparticles during the Oxygen Evolution Reaction. Angew. Chem. Int. Ed. 53, 7169-7172 (2014).

51. Youngblood, W. J. et al. Photoassisted overall water splitting in a visible lightabsorbing dye-sensitized photoelectrochemical cell. J. Am. Chem. Soc. 131, 926-927 (2009).

52. Lin, Y. J. et al. Semiconductor nanostructure-based photoelectrochemical water splitting: a brief review. Chem. Phys. Lett. 507, 209-215 (2011).

53. Nakagawa, T., Beasley, C. A. \& Murray, R. W. Efficient electro-oxidation of water near its reversible potential by a mesoporous $\mathrm{IrO}_{\mathrm{x}}$ nanoparticle film. J. Phys. Chem. C 113, 12958-12961 (2009).

54. Ouattara, L., Fierro, S., Frey, O., Koudelka, M. \& Comninellis, C. Electrochemical comparison of $\mathrm{IrO}_{2}$ prepared by anodic oxidation of pure iridium and $\mathrm{IrO}_{2}$ prepared by thermal decomposition of $\mathrm{H}_{2} \mathrm{IrCl}_{6}$ precursor solution. J. Appl. Electrochem. 39, 1361-1367 (2009).

55. Bard, A. J. \& Faulkner, L. R. Electrochemical Methods: Fundamentals and Applications 2nd edn (Wiley, 2001).

56. Morris, N. D., Suzuki, M. \& Mallouk, T. E. Kinetics of electron transfer and oxygen evolution in the reaction of $\left[\mathrm{Ru}(\mathrm{bpy})_{3}\right]^{3+}$ with colloidal iridium oxide. J. Phys. Chem. A 108, 9115-9119 (2004).

57. McCrory, C. C. L., Jung, S. H., Peters, J. C. \& Jaramillo, T. F. Benchmarking heterogeneous electrocatalysts for the oxygen evolution reaction. J. Am. Chem Soc. 135, 16977-16987 (2013).

58. Bediako, D. K., Costentin, C., Jones, E. C., Nocera, D. G. \& Saveant, J. M. Proton-electron transport and transfer in electrocatalytic films. application to a cobalt-based $\mathrm{O}_{2}$-evolution catalyst. J. Am. Chem. Soc. 135, 10492-10502 (2013).

59. Surendranath, Y., Kanan, M. W. \& Nocera, D. G. Mechanistic studies of the oxygen evolution reaction by a cobalt-phosphate catalyst at neutral pH. J. Am. Chem. Soc. 132, 16501-16509 (2010).

60. Scholten, M. \& Vandenmeerakker, J. E. A. M. On the mechanism of ITO etching-the specificity of halogen acids. J. Electrochem. Soc. 140, 471-475 (1993).

61. Kushner-Lenhoff, M. N., Blakemore, J. D., Schley, N. D., Crabtree, R. H. \& Brudvig, G. W. Effects of aqueous buffers on electrocatalytic water oxidation with an iridium oxide material electrodeposited in thin layers from an organometallic precursor. Dalton Trans. 42, 3617-3622 (2013).

62. Yagi, M., Tomita, E., Sakita, S., Kuwabara, T. \& Nagai, K. Self-assembly of active $\mathrm{IrO}_{2}$ colloid catalyst on an ITO electrode for efficient electrochemical water oxidation. J. Phys. Chem. B 109, 21489-21491 (2005).

63. Hara, M., Waraksa, C. C., Lean, J. T., Lewis, B. A. \& Mallouk, T. E. Photocatalytic water oxidation in a buffered tris (2,2'-bipyridyl)ruthenium complex-colloidal $\mathrm{IrO}_{2}$ system. J. Phys. Chem. A 104, 5275-5280 (2000).

64. Ahn, H. S., Yano, J. \& Tilley, T. D. Photocatalytic water oxidation by very small cobalt domains on a silica surface. Energy Environ. Sci. 6, 3080-3087 (2013).

65. Coperet, C., Chabanas, M., Saint-Arroman, R. P. \& Basset, J. M. Homogeneous and heterogeneous catalysis: bridging the gap through surface organometallic chemistry. Angew. Chem. Int. Ed. 42, 156-181 (2003).

66. Liu, J. et al. Oriented Nanostructures for Energy Conversion and Storage. ChemSusChem 1, 676-697 (2008).

67. Chen, Z. F. et al. Nonaqueous catalytic water oxidation. J. Am. Chem. Soc. 132, 17670-17673 (2010).

68. deKrafft, K. E. et al. Electrochemical water oxidation with carbon-grafted iridium complexes. ACS Appl. Mater. Interfaces 4, 608-613 (2012).

69. Smith, R. D. L., Sporinova, B., Fagan, R. D., Trudel, S. \& Berlinguette, C. P. Facile photochemical preparation of amorphous iridium oxide films for water oxidation catalysis. Chem. Mater. 26, 1654-1659 (2014).

70. McFarland, E. W. Solar energy: setting the economic bar from the top-down. Energy Environ. Sci. 7, 846-854 (2014).

\section{Acknowledgements}

This work was partially supported by the National Science Foundation (NSF MRSEC DMR 1119826), an NSF Graduate Research Fellowship (S.W.S.), the U.S. Department of Energy (DE-FG02-07ER15909, S.W.S. and C.A.S.) and both the Center for Catalytic Hydrocarbon Functionalization (CCHF) as well as the Argonne-Northwestern Solar Energy Research 
(ANSER) Center, Energy Frontier Research Centers funded by the U.S. Department of Energy, Numbers DE-SC0001298 (R.H.C.) and DE-SC0001059 (J.M.T., R.H.C. and G.W.B.), respectively. U.H. acknowledges the Alexander von Humboldt Foundation for a Feodor Lynen Research Fellowship at Yale and the Centre for Sustainable Chemical Technologies at the University of Bath (EPSRC grant no. EP/G03768X/1) for a Whorrod Research Fellowship. Facilities use was supported by the Yale Institute for Nanoscience and Quantum Engineering (YINQE) and Yale's Center for Research on Interface Structures and Phenomena (CRISP). We thank Dr Lior Kornblum, Marvin Wint, Dr Charles Ahn and Dr Fred Walker for access to XPS and assistance with data collection and analysis. Dr Jesús Campos, Jeffrey Chen, TengHooi Goh and Dr Andre Taylor are acknowledged for their assistance with electrode preparation, Dr Aaron Bloomfield for experimental assistance, Dr Christopher Koenigsmann for comments on electrochemistry and Dr David Vinyard for assistance with electron paramagnetic resonance experiments.

\section{Author contributions}

S.W.S. developed the concept of heterogenizing via direct binding the molecular homogeneous WOCs that were discovered previously by U.H., R.H.C. and G.W.B. S.W.S., J.M.T and U.H. designed the experiments, S.W.S. carried out the electrode preparation, and S.W.S. and J.M.T. performed the experiments. S.W.S. wrote the manuscript and supporting information with contributions from J.M.T. U.H., R.H.C., G.W.B. and C.A.S. revised and edited the manuscript before submission. U.H., R.H.C., G.W.B. and C.A.S. supervised the work

\section{Additional information}

Supplementary Information accompanies this paper at http://www.nature.com/ naturecommunications

Competing financial interests: US Patent Application Number 14/317,906, a patent application by S.W.S., U.H., J.M.T., G.W.B. and R.H.C, was previously filed for the intellectual property described in this article. C.A.S. declares no competing financial interest.

Reprints and permission information is available at http://npg.nature.com/ reprintsandpermissions/

How to cite this article: Sheehan, S. W. et al. A molecular catalyst for water oxidation that binds to metal oxide surfaces. Nat. Commun. 6:6469 doi: 10.1038/ncomms7469 (2015).

(c) (i) This work is licensed under a Creative Commons Attribution 4.0 International License. The images or other third party material in this article are included in the article's Creative Commons license, unless indicated otherwise in the credit line; if the material is not included under the Creative Commons license, users will need to obtain permission from the license holder to reproduce the material. To view a copy of this license, visit http://creativecommons.org/licenses/by/4.0/ 